\title{
Preliminary cost variance modeling to compare autologous intraovarian platelet-rich plasma vs. standard hormone replacement therapy for menopause management
}

\author{
E. Scott Sills $1,2, *$ and Seang Lin Tan ${ }^{3,4}$
}

${ }^{1}$ Reproductive Research Section, FertiGen/CAG; San Clemente, California USA

${ }^{2}$ Department of Obstetrics \& Gynecology, Palomar Medical Center; Escondido, California USA ${ }^{3}$ OriginElle Fertility Clinic; Montréal, Quebec CAN

${ }^{4}$ Department of Obstetrics \& Gynecology, McGill University Health Centre; Montréal, Quebec

CAN

* Correspondence: FertiGen/CAG P.O. Box 73910, San Clemente, California 92673 USA; email: ess@prp.md 표 +1 949-899-5686

\begin{abstract}
Background: Menopause symptoms and hormone replacement therapy (HRT) are among the most common reasons patients seek gynecological advice. Although at least half of all women in developed countries will take HRT during their lifetime, the treatment is not without risk and guidance on HRT is mixed. Greater awareness of negative HRT health effects from extended use has piqued interest in 'safer options'. Menopause reversal with autologous ovarian platelet-rich plasma (OPRP) has brought this restorative approach forward for consideration, but appropriateness and cost-effectiveness require examination. Methods: HRT and OPRP data from USA were projected to compare cumulative 1yr patient costs using stochastic Monte Carlo modeling. Results: Mean \pm SD cost-to-patient for HRT including initial consult plus pharmacy refills was estimated at about $\$ 576 \pm 246 / \mathrm{yr}$. While OPRP included no pharmacy component, an estimated 4 visits over $1 \mathrm{yr}$ for OPRP maintenance entailed ultrasound, phlebotomy/sample processing, surgery equipment, and incubation/laboratory expense, yielding mean $\pm \mathrm{SD}$ cost for OPRP at $\$ 8,710 \pm 4,911 /$ yr $(p<0.0001$ vs. HRT, by $t$-test). Upper-bound estimates for annual HRT and OPRP costs were $\$ 1,341$ and $\$ 22,232$, respectively. Conclusions: While HRT and OPRP may have similar efficacy and safety for menopause therapy, they diverge sharply in cost-effectiveness. Most patients would likely find OPRP too complex, invasive, and expensive to be competitive vs. HRT. Although OPRP is an interesting and cautiously useful technique for selected menopause patients reluctant to use HRT, repurposing this infertility treatment for wider use appears inefficient compared to standard HRT currently available.
\end{abstract}

Key words; menopause; alternative therapy; PRP; health spend 


\section{Running title: Ovarian PRP vs. HRT in menopause}

\section{Introduction}

Hormone replacement therapy (HRT) is an important part of menopause care. Without timely intervention, increased risks of cardiovascular disease, osteoporosis, premature cognitive decline and vaginal atrophy can lead to reduced quality-of-life in menopause (Marjoribanks et al, 2017; Thaung Zaw et al, 2018). Synthetic hormone usage in this population was on track for an ever-upward rise, until safety concerns from the Women's Health Initiative (WHI) emerged in 2002. The global HRT market swiftly collapsed when worrisome results became generally known (Donovitz, 2021), and promotional spending was cut particularly for those prescriptions most directly implicated in the WHI (Majumdar et al, 2004). National data showed diminished HRT sales affected all hormone products across every patient demographic (Hing \& Brett, 2006), although bettereducated patients seemed more inclined to consider alternatives to HRT compared to those with different educational backgrounds (Krzyżanowska \& Górecka, 2021).

This market disruption created an unexpected opening for compounded 'bioidentical hormones' (Vance, 2007; Santoro \& Liss, 2021) to grow their niche within an economy worth at least $\$ 20 \mathrm{~B}$. These alternative treatments exploited their exempt non-FDA-regulated status to cultivate an image of being safer than conventional HRT, achieving an outsized presence.

In 2016, an infertility treatment termed 'ovarian rejuvenation', unrelated to menopause care, was developed in Athens, Greece (Pantos et al, 2016). This innovation was a corollary to PRP work successfully applied in other clinical fields, but not previously known in a reproductive context. The technique involved minor surgery to insert autologous platelet-rich plasma (PRP) into ovarian tissue. Remarkably, case data and small series subsequently described healthy pregnancies and deliveries among poor-prognosis patients, including many initially thought to be menopausal (Sfakianoudis et al, 2018; Farimani et al, 2019; Pantos et al, 2019). Against this landscape, the possibility to refocus ovarian PRP (OPRP) as a way to address symptomatic menopause has drawn notice akin to other alternative therapies. The present research is the first to develop an economic model to assess OPRP use in this setting.

\section{PRP: Another 'bioidentical'?}

OPRP provides benefits congruent with other 'bioidentical' menopause treatments, but patient willingness to consider non-traditional options and altered physician prescribing patterns away from HRT are essential preconditions (Morris \& Currie, 2020). There has been a large feminist contribution to the sociomedical discourse, rightly rejecting tendencies to manage menopause as one homogenous condition (Chalouhi, 2017). The bespoke, personal dimension of OPRP assuages this discontent. One critique decried healthcare providers who continue to 'let patients down' with low value medical advice, harming not just the patient but also damaging the entire health economy (Cumming et al, 2015). But for menopause, is OPRP high value? 
As 'self-tissue' processed fresh prior to each use, autologous platelet-rich plasma derivatives carry intrinsic appeal as a natural, non-synthetic alternative to bulk HRT engineered at industrial scale. Hypersensitivity reactions are very rare, thought to occur only in response to platelet activators rather than the autologous PRP itself (Latalski et al, 2019). One study sampled preferences among patients (average age 60.5yrs) where compounded options were felt to be 'just as good or better' than manufactured pharmaceuticals, with $>95 \%$ satisfied with everything but the cost (McPherson et al, 2019).

The commercial rise of 'bioidenticals' has not been restrained by the lack of supporting evidence to prove they are safer or any more effective than HRT (Huntley, 2011). With OPRP, its chief weakness on evidence is that studies to date have not included a control/saline injection group. This could be relevant if simple mechanical stretching and/or local microtrauma to ovarian tissue triggers temporary inflammatory signaling and improved ovarian function (Atkinson et al, 2021). The assertion that 'ovarian acupuncture' could evoke meaningful AMH change was countered by finding platelet concentration, not mere injection itself, relates to $\mathrm{AMH}$ response after OPRP treatment (Sills et al, 2020a). Moreover, when such 'blank doses' were studied in an animal model, it was shown that saline injection alone had no effect on ovarian function (Ahmadian et al, 2020).

General agreement exists that any kind of HRT can reduce bone turnover, slow bone reabsorption, and increase bone mineral density (Cagnacci \& Venier, 2019), but extant literature on OPRP has not shown any such effect with this procedure. It is plausible OPRP could similarly protect against fracture risk by improving BMD, with the proviso 'ovarian rejuvenation' was maintained. This would parallel experience with HRT where evidence shows skeletal benefits are not retained after HRT stops (Rozenberg et al, 2020).

Most patients attending for OPRP expect to have both ovaries injected, but this is not always possible. In one series, unplanned unilateral access occurred in about $35 \%$ of cases (Sills et al, 2020a); this usually occurred when higher BMI and poor visibility blocked clear visualization of adnexal structures on ultrasound. Despite heavier patients being less likely to undergo bilateral ovarian injection, sub-group analysis showed injection of just one ovary had similar effects for improved overall sexual experience $(p=0.85)$, energy level $(p=0.42)$, and menses recovery $(p=0.15)$ compared with patients who received bilateral OPRP (Sills et al, 2019).

\section{Methods}

Several HRT routes of administration are available, although the two most popular are oral and transdermal (Allied Market Research, 2021) which were selected for this comparison. These main modes of HRT administration are followed by patient-applied topical creams or lotions, chiefly as intravaginal preparations. Conversely, OPRP delivery methods show almost no variation. All 'ovarian rejuvenation' relies on resource intensive needle insertion by ultrasound (TV-USG) guidance or laparoscopy to access ovarian tissue. A recent literature review (Sills \& Wood, 2021) suggests that laparoscopy is uncommonly used for OPRP, unless some prevailing 
circumstance renders the TV-USG approach unsuitable or unsafe. Accordingly, our calculation included TV-USG only for OPRP.

Since OPRP shares functional overlap with in vitro fertilization (IVF) and ovarian cyst aspiration, related audits (Masler \& Strickland, 2013) were used to guide estimates for OPRP, especially for initial doctor's consult. Economic data were obtained from aggregate U.S. medical charge surveys (Allied Market Research, 2021) on single-use surgical equipment and reagents, incubator, laboratory and technical staff costs, as well as published pharmacy estimates for representative prescribed HRT agents (as out-of-pocket cost). To isolate annual estimates for the treatments under study, the model excluded costs incurred outside menopause symptom management.

Comparisons between patient medical cost typically relies on National Inpatient Sample data where cost-to-charge ratios are calculated from Healthcare Cost \& Utilization Project figures (Sheyn et al, 2017). But because OPRP is an out-patient procedure and HRT is medication only, for this analysis cost variance (CVAR) was defined as follows:

$$
\mathrm{C}_{\mathrm{VAR}}=\mathrm{V}_{e}-\mathrm{C}_{a} \text {, }
$$

where:

$$
\begin{gathered}
\mathrm{V}_{e}=\text { treatment benefit (earned value) } \\
\mathrm{C}_{a}=\text { actual cost. }
\end{gathered}
$$

Due to confined data with both treatments (especially OPRP) costs were considered stochastic to test net effect on individual patient expenditure. Probabilistic modeling can help reduce epistemic uncertainty when partial data are prevalent. This effect was simulated by Monte Carlo simulations $(n=500)$ iteratively to generate forward modeling. For HRT, input distributions were defined by past and current pharmacy costs, formulaic assumptions included accepting at least one of the current HRT preparations as capable to offer high (or at least adequate) Ve to consumers (patients). This is probably correct given industry data and academic literature indicating a low drop-out rate for HRT (Samsioe et al, 2007; Chang et al, 2021). In contrast, OPRP is a new arrival with no longitudinal information available on compliance and satisfaction. Early monitoring suggests complications/adverse events with OPRP may be lower than for HRT, so this model considered treatment efficacy as comparable across the two methods studied. Self-pay HRT cost to patient was thus calculated from published pharmacy data to supply product refills for one year, where monthly mean $\pm S D=\$ 47.50 \pm 20$. Projecting patient cost for OPRP was by itemizing its two main components (specimen processing + office surgery) which were unbundled, costed separately then recombined, where mean $\pm \mathrm{SD}=$ $\$ 8750 \pm 5000$. Results were annualized by stochastic analysis using ASTM E1369 v.1.0 (National Institute of Standards \&Technology; Gaithersburg, MD), on Microsoft Excel.

\section{Results}

Using available published public datasets for office visit costs, administrative and associated laboratory fees, as well as out-of-pocket cost for non-generic HRT, yearly 2021 patient cost was projected at approximately $\$ 576 \pm 238$, with maximum annual HRT spend estimated at $\$ 1340$ (see Figure 
1). OPRP cost was captured by disaggregating individual elements and then adding the constituent parts to calculate total patient expense. This comprised PRP processing and preparation (CPT 0232T) which was associated with a historic cost per injection of $\$ 2,000$ (Storrs, 2009). Since that cost was published in 2009, inflation adjustment (U.S. Inflation Calculator, 2021) corrected the per-injection value to current (2021) equivalency, approximately $\$ 2,557$. Thus, four PRP samples prepared over 1yr would be about $\$ 10,000$. The surgical component of OPRP shares equipment and technical overlays with standard ovarian cyst drainage by ultrasound guidance (CPT 58800), where average cost is $\$ 1,875$. Experience with OPRP suggests baseline/initial injection appointments require more procedure time, but as ovaries respond (enlarge) in later months the technical difficulty is reduced, using less clinic time. However, this trend is tentative and derived from one California center only, so uniform visit times were used for each visit. This produces a projected cost of $\$ 7,500$ for planned OPRP series over $1 y r$. To account for geographic pricing differences the sum of the two main cost centers was split (50\%) and a large \pm SD of 5000 was factored to account for regional fluctuation. Consequently, the annual 2021 patient cost for OPRP was estimated at approximately $\$ 8,710 \pm 4,911$ ( $p<0.0001$ vs. HRT, by Student's $t$-test), with maximum 4-visit patient cost projected at \$22,232.

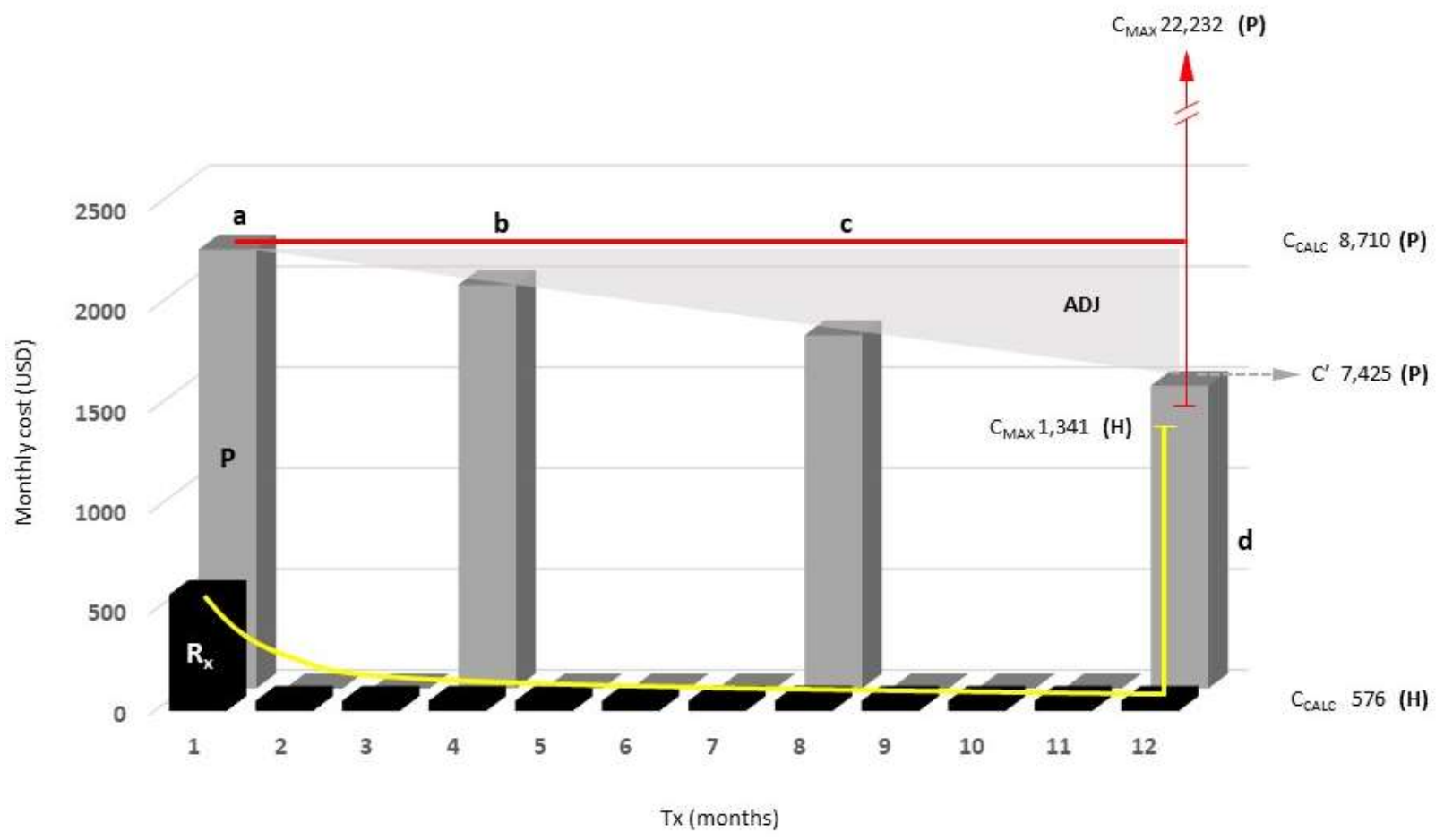

Figure 1. Projected annual costs in USD for two menopause treatments, standard (Rx) hormone replacement therapy and ovarian platelet-rich plasma (P) estimated by Monte Carlo simulations ( $n=500)$. Cumulative spend (yellow line vs. red line) for HRT was significantly below OPRP $(p<0.0001$, by Student's $t$-test) and persisted $\left(C^{\prime}\right)$ after adjustment (ADJ) for shorter-duration OPRP clinic visits (b,c,d). Maximum costs (CMAX) for HRT and OPRP were modeled at \$1,341 and \$22,232, respectively.

\section{Discussion}


Each year, about two million women enter menopause and about $40 \%$ are likely to use 'bioidentical hormones' to help manage their symptoms (Donovitz, 2021). No research has focused on OPRP use specifically in this population and what ancillary data are available usually appear without reference to fiscal parameters. Menopause-associated estrogen deficiency is intrinsically subjective, but validated questionnaires can monitor several relevant parameters during this phase (Sourouni et al, 2021). There is a nascent but enlarging body of OPRP literature investigating these topics. Genitourinary irritation, vaginal atrophy or dryness, and/or dyspareunia have a deeply personal and negative effect on women's health and relationships, impacting at least half of all menopausal women (Marino, 2021). When these symptoms were queried after OPRP, slightly more than half $(41 / 80)$ noted improved vaginal lubrication/cervical mucus production with treatment (Sills et al, 2019). Elevated arousal/sexual desire was similarly reported by study patients following OPRP, including ability to achieve orgasm/climax during sex (noted in $45 \%$ of patients). The report failed to speculate why patients with improved overall sexual experience were significantly older than those reporting no change ( $47.9 \pm 6.3$ vs. $43.5 \pm 5.2 \mathrm{yrs}$; $p=0.001$ ). Unfortunately, the study was also not designed to rank or sequence these improvements, or measure the duration of their perceived effects (Sills et al, 2019).

Perimenopause is frequently accompanied by increased fatigue (Kalmbach et al, 2019; Chiu et al, 2021) and self-reported 'low energy'. How effectively this problem was managed by an 'ovarian rejuvenation' technique was assessed using a confidential questionnaire in 2019. Regarding daily average energy level, 45 of 80 (56.3\%) experienced less fatigue after OPRP (Sills et al, 2019). Data were also collected on skin texture/tone, nail growth, and scalp hair thickness/volume after receiving OPRP. These dermatologyrelated parameters were tabulated together, and 37/80 patients $(46.3 \%)$ registered cosmetic improvements after treatment, a change closely correlated with daily average energy level (Pearson's $r=0.41 ; p<0.001$ ) (Sills et al, 2019).

Estrogens modulate serotonin and noradrenalin balance, thereby influencing cognitive function and mood (Birkhäuser, 2021). HRT in menopause is believed to lower dementia risk if implemented before symptoms begin, and thus may be safely prescribed early for women free of dementia (Petra et al, 2021). Authors have reported conflicting data on HRT effects on cognition (Conde et al, 2021) and for OPRP it remains to be shown if this may provide useful corrections in mood, affect, or cognition. A pilot program was used to measure cognitive acuity and mentation after OPRP, with $37 / 80$ patients $(46.3 \%)$ experiencing substantially less 'brain fog' after the procedure (Sills et al, 2019). Of note, improved cognitive acuity was significantly correlated with skin improvements $(r=0.36 ; p<0.01)$ and energy level $(r=0.47 ; p<0.001)$. For sleep quality, $43.8 \%$ of study subjects reported this was better after OPRP. Sleep optimization often accompanied skin improvements $(r=0.42 ; p<0.01)$, increased energy level $(r=0.42 ; p<0.01)$, and better mentation $(r=0.39 ; p<0.01)$ (Sills et al, 2019).

While sleep quality, daytime fatigue, and workplace productivity share connections, precise quantification of relevant factors is complex and surprisingly difficult to study. Detailed research (Kagan et al, 2021) reported 
unemployment risk to be $>30 \%$ higher for women with vs. without new-onset sleep disturbances. For women age 42-64 in USA, annual lost productivity attributed to sleep disorders exceeded \$2B (Kagan et al, 2021).

Greek experts (Sfakianoudis et al, 2020) were able to reverse menopause in more than half (18/30) of participants receiving 'ovarian rejuvenation', and also documented significant improvements in antral follicle count and serum AMH. In this population of non-HRT users between ages 45-55yrs, participants had no menses for at least 12 months before OPRP. Improved hormonal levels were verified in 24/30 patients after intraovarian PRP (Sfakianoudis et al, 2020). This aligns with data on 80 California patients (mean \pm SD age $45.5 \pm 6 y r s$ ) where more than half had irregular or absent menses at baseline (Sills et al, 2019). While return of menstruation or recovery of regular menses was attained by $24 \%$ after OPRP, among those using HRT before enrollment, most (31/46) were able to stop all hormone use following OPRP. For sexually active patients ( $86 \%$ ) only three reported reduced sexual activity after OPRP $(4.4 \%)$. It was also possible to assess improvement $(27.3 \%)$ vs. impairment $(4.4 \%)$ in sexual activity after 'ovarian rejuvenation', and the difference favoring OPRP was highly significant ( $p=0.008$, by N-1 $\chi 2$ test). For the 11 women not sexually active before OPRP, return of sexual activity after treatment was reported by $>25 \%$. While no patient reported weight gain with OPRP, weight loss among patients completing OPRP was small but significant (Sills et al, 2019). Vasomotor symptoms were also evaluated following OPRP, with significant improvement in 'hot flashes' reported 14wks after treatment (Merhi et al, 2021).

Our audit of menopausal OPRP use has at least two limitations: 1) Provisional calculations from statistical modeling can be eclipsed by clinical/actuarial data (when available), and 2) Published 'ovarian rejuvenation' protocols show that platelet activation and specimen processing remain to be harmonized. Neither problem confronts HRT, and indeed some common elements exist which make comparison easier. For example, the basic clinic appointment required to obtain either treatment (HRT or OPRP) is the same, between $\$ 75-\$ 200$. And before determining any treatment, the physician is likely to perform an examination and obtain tests to assess hormone levels (HRT Cost, 2021).

It should be noted that many U.S. health insurance plans include at least one HRT option on formulary, but sometimes cover is refused on the basis that treating menopause is a personal choice-using a taxonomy where menopause is not a true disease state, but rather 'a normal part of aging' (HRT Cost, 2021). Not every patient responds to HRT satisfactorily and this is surely true for OPRP, as well. Surprisingly little is known about the proportion of refractory HRT cases although the menu of available HRT delivery systems disfavors OPRP - which, for now, is essentially nondiversified. A condensed plasma cytokine PRP variant has been successfully used for ovaries (Sills et al, 2020b) but not specifically for menopause, so more research is needed.

Because OPRP is a much newer treatment modality, it suffers from wide cost fluctuations commonly seen with novelty procedures. But as efficiencies improve, it is possible OPRP costs will stabilize at lower levels to bring the treatment within reach for more patients. The difference between HRT and OPRP dose schedule is also noteworthy: Months can pass between 'ovarian 
rejuvenation' visits whereas HRT must be replaced/refilled on a monthly, weekly, or every-other-day basis. The 4-month dosing pattern used here was based on feedback shared by California patients; lasting effects of OPRP could be more durable and allow less frequent 'booster' visits. Yet, any advantage from eliminating HRT pharmacy refills is obviated (dwarfed, even) by the dramatically higher cost of OPRP, even if the latter involved only a single yearly visit. And because some clinics provide 'ovarian rejuvenation' with anesthesia - a variable our formula omitted - OPRP could be even more expensive than estimated here.

Consumer preference for 'bioidenticals' shows how dissatisfaction can drive new demand, even when the new item costs more. Perhaps the simplest explanation for the superiority of HRT identified here is the crushing inefficiency of OPRP when structured as a 'subscription service'. Success with OPRP has showcased its role in ovarian reserve upticks, over months or weeks, to optimize response to gonadotropin treatments for IVF. When this enables unassisted pregnancy (Sfakianoudis et al, 2020; Petryk \& Petryk, 2020; Sills et al, 2020b), the investment in OPRP is recompensed by obviating costs of IVF. Quality-of-life gains or menopause reversal after OPRP while awaiting IVF, although welcome and never trivial, are best marked as incidental objectives. Thus, repurposing OPRP for nonreproductive use appears workable only if its significantly higher cost brings consistent benefits not attainable with simpler and cheaper HRT. Improved ovarian capacity, sexual health, and metabolism with OPRP may suggest limited applications beyond fertility practice, but the commercial dominance of standard HRT seems secure. The convenience and low cost of HRT will be difficult, if not impossible, for OPRP to overcome. The subject warrants further monitoring given the ever-growing number of menopausal patients.

Authors' contributions: ESS developed the project, ESS and SLT jointly reworked drafts, and both approved the final manuscript.

Disclosure statement: ESS has been issued a provisional U.S. patent for process \& treatment of ovarian disorders using platelet cytokine derivatives.

\section{References:}

Ahmadian, S., Sheshpari, S., Pazhang, M., Bedate, A.M., Beheshti, R., Abbasi, M.M., Nouri, M., Rahbarghazi, R., Mahdipour. M. (2020). Intra-ovarian injection of platelet-rich plasma into ovarian tissue promoted rejuvenation in the rat model of premature ovarian insufficiency and restored ovulation rate via angiogenesis modulation. Reproductive Biology \& Endocrinology, 18, 1-13. https://doi.org/10.1186/s12958-020-00638-4

Allied Market Research. 2021 HRT market analysis:1-262. https://www.alliedmarketresearch.com/hormonereplacement-therapy-market-A09508 [file accessed 15 Oct 2021].

Atkinson L., Martin F., Sturmey R.G. (2021). Intraovarian injection of platelet-rich plasma in assisted reproduction: too much too soon? Human Reproduction, 36(7), 1737-1750. https://doi.org/10.1093/humrep/deab106

Birkhäuser M. (2021). Climacteric depression and anxiety. Therapeutische Umschau, 78(8), 427-434. https://doi.org/10.1024/0040-5930/a001293

Cagnacci A., Venier M. (2019). The controversial history of hormone replacement therapy. Medicina (Kaunas), 55(9), 602. https://doi.org/10.3390/medicina55090602

Chalouhi S. (2017). Menopause: A complex and controversial journey. Post Reproductive Health, 23(3), 128-131. https://doi.org/10.1177/2053369117711346

Chang C.Y., Tsai F.J., Chiou J.S., Chiu M.L., Lin T.H., Liao C.C., Huang, S.M., Liang, W.M., Lin, Y.J. (2021). Timing and dosage of and adherence to hormone replacement therapy and fracture risk in women with menopausal syndrome in Taiwan: A nested case-control study. Maturitas, 146, 1-8. https://doi.org/10.1016/j.maturitas.2020.12.010 
Chiu, H.H., Tsao, L.I., Liu, C.Y., Lu, Y.Y., Shih, W.M., Wang, P.H. (2021). The perimenopausal fatigue selfmanagement scale is suitable for evaluating perimenopausal Taiwanese women's vulnerability to fatigue syndrome. Healthcare (Basel), 9(3), 336. https://doi.org/10.3390/healthcare9030336

Conde, D.M., Verdade, R.C,, Valadares, A.L.R., Mella, L.F.B., Pedro, A.O., Costa-Paiva, L. (2021). Menopause and cognitive impairment: A narrative review of current knowledge. World Journal of Psychiatry, 11(8), 412-428. https://doi.org/10.5498/wjp.v11.i8.412

Cumming, G.P., Currie, H., Morris, E., Moncur, R., Lee, A.J. (2015). The need to do better - Are we still letting our patients down and at what cost? Post Reproductive Health, 21(2), 56-62. https://doi.org/10.1177/2053369115586122

Donovitz, G.S. (2021). Society position statements on bio-identical hormones-Misinformation leads to a dilemma in women's health. Healthcare (Basel), 9(7), 782. https://doi.org/10.3390/healthcare9070782

Farimani, M., Heshmati, S., Poorolajal, J., Bahmanzadeh, M. (2019). A report on three live births in women with poor ovarian response following intra-ovarian injection of platelet-rich plasma (PRP). Molecular Biology Reports, 46(2), 1611-1616. https://doi.org/10.1007/s11033-019-04609-w

Hing, E., Brett, K.M. (2006). Changes in U.S. prescribing patterns of menopausal hormone therapy, 2001-2003. Obstetrics \& Gynecology, 108(1), 33-40. https://doi.org/10.1097/01.AOG.0000220502.77153.5a

Hormone replacement therapy cost. (2021) Cost Helper Health (online patient education resource). https://health.costhelper.com/hormone-replacement-therapy.html\#extres2 [file accessed 15 Oct 2021].

Huntley, A.L. (2011). Compounded or confused? Bioidentical hormones and menopausal health. Menopause International, 17(1), 16-18. https://doi.org/10.1258/mi.2011.011009

Kagan, R., Shiozawa, A., Epstein, A.J., Espinosa, R. (2021). Impact of sleep disturbances on employment and work productivity among midlife women in the US SWAN database: a brief report. Menopause, 28(10), 1176-1180. https://doi.org/10.1097/GME.0000000000001834

Kalmbach, D.A., Cheng, P., Arnedt, J.T., Cuamatzi-Castelan, A., Atkinson, R.L., Fellman-Couture, C., Roehrs, T., Drake, C.L. (2019). Improving daytime functioning, work performance, and quality of life in postmenopausal women with insomnia: Comparing cognitive behavioral therapy for insomnia, sleep restriction therapy, and sleep hygiene education. Journal of Clinical Sleep Medicine, 15(7), 999-1010. https://doi.org/10.5664/jcsm.7882

Krzyżanowska, M., Górecka, K. (2021). Women's knowledge on the menopausal transition in relation to their socioeconomic status. Przeglad Menopauzalny, 20(2), 81-87. https://doi.org/10.5114/pm.2021.106891

Latalski, M., Walczyk, A., Fatyga, M., Rutz, E., Szponder, T., Bielecki, T., Danielewicz, A. (2019). Allergic reaction to platelet-rich plasma (PRP): Case report. Medicine (Baltimore), 98(10), e14702. https://doi.org/10.1097/MD.0000000000014702

Majumdar, S.R., Almasi, E.A., Stafford, R.S. (2004). Promotion and prescribing of hormone therapy after report of harm by the Women's Health Initiative. JAMA, 292(16), 1983-1988. https://doi.org/10.1001/jama.292.16.1983

Marino, J.M. (2021). Genitourinary syndrome of menopause. Journal of Midwifery \& Women's Health, https://doi.org/10.1111/jmwh.13277 [online ahead of print]

Marjoribanks, J., Farquhar, C., Roberts, H., Lethaby, A., Lee, J. (2017). Long-term hormone therapy for perimenopausal and postmenopausal women. Cochrane Database of Systematic Reviews, 1(1), CD004143. https://doi.org/10.1002/14651858.CD004143.pub5

Masler, S., Strickland, R.R. (2013). The role of management in an in vitro fertilization practice. Seminars in Reproductive Medicine, 31(3), 204-210. https://doi.org/10.1055/s-0033-1336601

McPherson, T., Fontane, P., Bilger, R. (2019). Patient experiences with compounded medications. Journal of the American Pharmacy Association (2003), 59(5), 670-677.e2. https://doi.org/10.1016/j.japh.2019.05.005

Merhi, Z., Seckin, S., Mouanness, M. (2021). Intraovarian PRP injection improved hot flashes in a woman with very low ovarian reserve. Reproductive Sciences, https://ink.springer.com/article/10.1007\%2Fs43032-021-00655-7 [online ahead of print].

Morris, E., Currie, H. (2020). UK HRT shortages, how can this be good for anybody? Post Reproductive Health, 26(1), 3-4. https://doi.org/10.1177/2053369120910693

Pantos, K., Nitsos, N., Kokkali, G., Vaxevanoglou, T., Markomichali, C., Pantou, A., Grammatis, M., Lazaros, L., Sfakianoudis, K. (2016). Ovarian rejuvenation and folliculogenesis reactivation in peri-menopausal women after autologous platelet-rich plasma treatment [conference abstract]. Hum Reprod (Suppl): P-401. https://sa1s3.patientpop.com/assets/docs/111052.pdf

Pantos, K., Simopoulou, M., Pantou, A., Rapani, A., Tsioulou, P., Nitsos, N., Syrkos, S., Pappas, A., Koutsilieris, M., Sfakianoudis, K. (2019). A case series on natural conceptions resulting in ongoing pregnancies in menopausal and prematurely menopausal women following platelet-rich plasma treatment. Cell Transplantation, 28(9-10), 1333-1340. https://doi.org/10.1177/0963689719859539

Petra S, Johanna W, Anne-Sophie K, Christina G, Wiebke W, Heidrun J, Sabrina, B. (2021). Cognitive health after menopause: Does menopausal hormone therapy affect it? Best Practice \& Research, Clinical Endocrinology \& Metabolism, https://doi.org/10.1016/j.beem.2021.101565 
Petryk, N., Petryk, M. (2020). Ovarian rejuvenation through platelet-rich autologous plasma (PRP) - A chance to have a baby without donor eggs, improving the life quality of women suffering from early menopause without synthetic hormonal treatment. Reproductive Sciences, 27(11), 1975-1982. https://doi.org/10.1007/s43032-02000266-8

Rozenberg, S., Al-Daghri, N., Aubertin-Leheudre, M., Brandi, M.L., Cano, A., Collins, P., Cooper, C., Genazzani, A.R., Hillard, T., Kanis, J.A., Kaufman, J.M., Lambrinoudaki, I., Laslop, A., McCloskey, E., Palacios, S., PrietoAlhambra, D., Reginster, J.Y., Rizzoli, R., Rosano, G., Trémollieres, F., Harvey, N.C. (2020). Is there a role for menopausal hormone therapy in the management of postmenopausal osteoporosis? Osteoporosis International, 31(12), 2271-2286. https://doi.org/10.1007/s00198-020-05497-8

Samsioe, G., Dvorak, V., Genazzani, A.R., Hamann, B., Heikkinen, J., Mueck, A.O., Suzin, J., Kawakami, F.T., Ferreira, A., Sun, D., Arguinzoniz, M. (2007). One-year endometrial safety evaluation of a continuous combined transdermal matrix patch delivering low-dose estradiol-norethisterone acetate in postmenopausal women. Maturitas, 57(2), 171-181. https://doi.org/10.1016/j.maturitas.2007.01.001

Santoro, N., Liss, J. (2021). Compounded bioidentical hormones: Myths and realities. Clinical Obstetrics \& Gynecology, https://doi.org/10.1097/GRF.0000000000000650 [online ahead of print]

Sfakianoudis, K., Simopoulou, M., Grigoriadis, S., Pantou, A., Tsioulou, P., Maziotis, E., Rapani, A., Giannelou, P., Nitsos, N., Kokkali, G., Koutsilieris, M., Pantos, K. (2020). Reactivating ovarian function through autologous platelet-rich plasma intraovarian infusion: Pilot data on premature ovarian insufficiency, perimenopausal, menopausal, and poor responder women. Journal of Clinical Medicine, 9(6), 1809. https://doi.org/10.3390/jcm9061809

Sfakianoudis, K., Simopoulou, M., Nitsos, N., Rapani, A., Pappas, A., Pantou, A., Chronopoulou, M., Deligeoroglou, E., Koutsilieris, M., Pantos, K. (2018). Autologous platelet-rich plasma treatment enables pregnancy for a woman in premature menopause. Journal of Clinical Medicine, 8(1), 1. https://doi.org/10.3390/jcm8010001

Sheyn, D., Mahajan, S., Billow, M., Fleary, A., Hayashi, E., El-Nashar, S.A. (2017). Geographic variance of cost associated with hysterectomy. Obstetrics \& Gynecology, $\quad 129(5), \quad 844-853$. https://doi.org/10.1097/AOG.0000000000001966

Sills, E.S., Li, X., Rickers, N.S., Wood, S.H., Palermo, G.D. (2019). Metabolic and neurobehavioral response following intraovarian administration of autologous activated platelet rich plasma: First qualitative data. $\begin{array}{lllll}\text { Neuroendocrinology } & \text { Letters, } & 39(6), & 427-433 . & \text { PMID: }\end{array}$ https://www.nel.edu/userfiles/articlesnew/1553033179 39 6_sills_427-pdf.pdf

Sills, E.S., Rickers, N.S., Petersen, J.L., Li, X., Wood, S.H. (2020a). Regenerative effect of intraovarian injection of autologous platelet rich plasma: Serum anti-Mullerian hormone levels measured among poor-prognosis in vitro fertilization patients. International Journal of Regenerative Medicine, 3, 1-5. http://dx.doi.org/10.31487/j.RGM.2020.01.02

Sills, E.S., Rickers, N.S., Wood, S.H. (2020b). Intraovarian insertion of autologous platelet growth factors as cell-free concentrate: Fertility recovery and first unassisted conception with term delivery at age over 40. International Journal of Reproductive Biomedicine, 18(12), 1081-1086. https://doi.org/10.18502/ijrm.v18i12.8030

Sills, E.S., Wood, S.H. (2021 - in press). Progress in human ovarian rejuvenation: Current platelet-rich plasma and condensed cytokine research activity by scope and international origin. Clinical \& Experimental Reproductive Medicine, pending

Sourouni, M., Zangger, M., Honermann, L., Foth, D., Stute, P. (2021). Assessment of the climacteric syndrome: a narrative review. Archives of Gynecology \& Obstetrics, 304(4), 855-862. https://doi.org/10.1007/s00404-02106139-y

Storrs, C. (2009). Is platelet-rich plasma an effective healing therapy? Scientific American, 18 December https://www.scientificamerican.com/article/platelet-rich-plasma-therapy-dennis-cardone-sports-medicineinjury/ [file accessed 15 Oct 2021].

Thaung Zaw, J.J., Howe, P.R.C., Wong, R.H.X. (2018). Postmenopausal health interventions: Time to move on from the Women's Health Initiative? Ageing Research Reviews, 48, 79-86. https://doi.org/10.1016/j.arr.2018.10.005

U.S. inflation calculator. (2021). https://www.usinflationcalculator.com/ [site accessed 15 Oct 2021].

Vance, D.A. (2007). Premarin: The intriguing history of a controversial drug. International Journal of Pharmaceutical Compounding, 11(4), 282-286. https://ijpc.com/Abstracts/Abstract.cfm?ABS=2619 